Génét. Sél. Evol., 1986, 18 (3), 249-260

\title{
Evolution de l'effectif génétique de lignées de Drosophila melanogaster soumises à une sélection artificielle
}

\author{
Araceli GALLEGO et Aurora GARCÍA-DORADO \\ Departamento de Genética, Facultad de Biología, \\ Universidad Complutense de Madrid, 28040 Madrid, Espagne
}

\section{Résumé}

On considère l'évolution de l'effectif génétique de lignées de Drosophila melanogaster soumises à une sélection individuelle pour diminuer le nombre de soies sternopleurales. L'effectif génétique moyen, estimé à partir des généalogies (JAMES, 1962), correspond à celui attendu sous l'effet de la sélection (ROBERTSON, 1961; BuRRows, 1984) pendant les 2 premières générations de sélection seulement, période où l'héritabilité est significativement différente de zéro. L'analyse des pourcentages de gènes apportés par chaque famille dans les générations successives (JAMES \& MCBRIDE, 1958) confirme que les lignées atteignent la fixation du caractère sélectionné dès la $2^{\mathrm{e}}$ génération. On conclut que la variabilité génétique du nombre de soies sternopleurales dans la population initiale est contrôlée par un petit nombre de gènes à effet important et à fréquence intermédiaire (GALlego \& LóPEZ-Fanjul, 1983).

Mots clés : Effectif génétique, sélection artificielle, Drosophila melanogaster.

\section{Summary \\ Evolution of effective population size in artificially selected lines of Drosophila melanogaster}

The evolution of effective population size in lines of Drosophila melanogaster individually selected for low sternopleural bristle number has been analysed. The pooled effective size estimated from pedigrees (JAMES, 1962) was in agreement with its expected value under selection (ROBERTSON, 1961 ; BURROWs, 1984) only for the first 2 generations of selection, the period where the heritability of the trait was significantly larger than zero. Estimates of percentages of genes contributed by each family to the next generation (JAMES \& MCBRIDE, 1958) confirm that the lines essentially reached fixation for the selected trait after 2 generations of selection. It is concluded that the genetic variation of sternopleural bristle number in the base population is due to segregation of only a few loci with large effects, at intermediate frequencies (GALlego \& LóPEZ-FANJUL, 1983).

Key words : Effective population size, artificial selection, Drosophila melanogaster. 


\section{Introduction}

La réponse à la sélection artificielle dans des populations d'effectif limité est influencée de plusieurs façons par la dérive génétique, dont l'effet est fonction de l'effectif génétique correspondant $(\mathrm{Ne})$. La valeur de ce paramètre est nécessaire pour calculer les valeurs espérées de la variabilité de la réponse (HILl, 1974 et 1977), la durée de la période pendant laquelle la réponse est linéaire (HiLL, 1977) et l'amplitude de la réponse totale (Robertson, 1960 ; Hill, 1985). De plus, l'augmentation de la consanguinité occasionne une diminution de la valeur sélective moyenne due à l'accumulation de gènes délétères. En outre, si le caractère est soumis à des effets de dominance, la valeur de la moyenne phénotypique est modifiée par l'effet de la consanguinité.

On trouve dans la bibliographie sur ce sujet plusieurs modèles qui permettent de calculer l'effectif génétique d'une lignée soumise à une sélection artificielle (JAMES, 1962 ; RoBERTSON, 1961; BuRrows, 1984); ces modèles se rapportent seulement à des gènes neutres, c'est-à-dire des gènes qui ne sont en relation ni avec le critère de sélection ni avec la valeur sélective des individus.

Beaucoup d'expériences sont conçues de façon à connaître les généalogies des individus qui permettent de calculer l'effectif génétique d'une lignée en utilisant la technique des "pourcentages de gènes" (JAMES \& MCBRIDE, 1958 ; JAMES, 1962). JAMES (1962) établit une relation entre la variance du changement de fréquence génique, et celle des pourcentages de gènes apportés à la génération $t$ par les mâles et les femelles qui appartiennent à la génération $t-1$; ainsi, cette équation permet d'estimer l'effectif génétique dans ce genre d'expérience.

D'autre part, en l'absence de généalogies, on peut prédire l'effectif génétique d'une lignée si on connaît le nombre de parents de chaque sexe et la distribution théorique du nombre de descendants par famille. Cependant, RoBERTson (1961) a démontré théoriquement que la sélection provoque une diminution de l'effectif génétique par rapport à celui calculé de cette façon, parce qu'elle favorise la contribution de certaines familles pour former le groupe sélectionné.

Les résultats analysés dans cet article proviennent d'un groupe de lignées sélectionnées individuellement pour la diminution du nombre de soies sternopleurales. Le comportement de ces lignées, analysé dans un article précédent (GALlEGo \& LópezFanjul, 1983) indique que la variation du caractère sélectionné est contrôlée par un petit nombre de gènes avec un effet tel qu'ils sont fixés rapidement. Ce sont les mêmes allèles qui sont fixés dans toutes les lignées.

Cette expérience permet d'analyser l'effet du processus de fixation sur l'évolution de l'effectif génétique des lignées. L'efficacité des différentes équations pour estimer la valeur de l'effectif génétique dans cette situation expérimentale sera vérifiée. De plus, il est possible de trouver un critère pour décider à partir de quel moment on peut dire que le caractère sélectionné est fixé, au moins pour la plupart des gènes. 


\section{Matériel et méthodes}

Tous les aspects du protocole expérimental sont détaillés dans un article précédent (Gallego \& Lófez-Fanjul, 1983). Nous utilisons la population Dahomey de Drosophila melanogaster. Le caractère sélectionné est le nombre total de soies sternopleurales. On calcule l'héritabilité du caractère sélectionné dans la population Dahomey suivant la méthode de Hill (1971). La valeur obtenue est de 0,6 $\pm 0,04$.

On sélectionne 6 lignées, chacune étant formée à chaque génération par 6 familles (chaque famille est composée d'individus issus d'un même couple) comportant chacune 10 individus de chaque sexe. On choisit comme géniteurs 6 mâles et 6 femelles dont le nombre de soies est le plus bas à chaque génération.

Les individus sélectionnés sont accouplés individuellement et au hasard dans chaque lignée. L'expérience est arrêtée à la $17^{\mathrm{e}}$ génération alors que les lignées ne répondent plus à la sélection depuis plusieurs générations.

On calcule l'effectif génétique (Ne) en utilisant l'équation de JAmEs (1962) :

$$
1 / \mathrm{Ne}=1 / 8 \mathrm{M}+1 / 8 \mathrm{~F}+\mathrm{m} \mathrm{V}(\mathrm{Ps})+\mathrm{f} V(\mathrm{Pd})
$$

$M$ et $F$ étant, respectivement, le nombre de mâles et femelles sélectionnés et $V$ ( $P s)$ et $V(P d)$ les variances des proportions de gènes apportés à la génération suivante par les mâles et les femelles, respectivement. Dans notre expérience les individus sélectionnés sont accouplés individuellement donc :

$$
\begin{aligned}
& \mathrm{M}=\mathrm{F}=\mathrm{N} / 2 \text { et } \mathrm{V}(\mathrm{Ps})=\mathrm{V}(\mathrm{Pd})=\mathrm{V}(\mathrm{P}) \text {; par ailleurs : } \\
& \quad \mathrm{V}(\mathrm{P})=\frac{\mathrm{V}(\mathrm{m})}{16(\mathrm{~N} / 2)^{2}}+\frac{\mathrm{V}(\mathrm{h})}{16(\mathrm{~N} / 2)^{2}}+\frac{2 \operatorname{cov}(\mathrm{m}, \mathrm{h})}{16(\mathrm{~N} / 2)(\mathrm{N} / 2)}=\mathrm{V}(\mathrm{k}) / 4 \mathrm{~N}^{2}
\end{aligned}
$$

$m$ et $f$ étant, respectivement, les nombres de mâles et de femelles sélectionnés dans chaque famille $(\mathrm{m}+\mathrm{h}=\mathrm{k})$ et $\mathrm{V}(\mathrm{k})$ la variance des contributions familiales au groupe sélectionné. L'équation (1) devient :

$$
\mathrm{N} / \mathrm{Ne}=(2+\mathrm{V}(\mathrm{k})) / 4
$$

Cette équation simplifiée est identique à celle obtenue par Wright (1940) et Crow (1954). (1961) :

On peut estimer l'effectif génétique $\left(\mathrm{Ne}^{v}\right)$ en utilisant l'équation de RoBERTSON

$$
\mathrm{N} / \mathrm{Ne}^{v}=(1-1 / 2 \mathrm{n})+(1-1 / \mathrm{n}) \mathrm{Z}_{\mathrm{t}}^{2} \mathrm{i}^{2} \mathrm{r}_{\mathrm{p}}
$$

$n$ étant le nombre d'individus de chaque sexe évalués par famille, $i$ l'intensité de sélection, $r_{p}$ la corrélation phénotypique intra-classe entre frères et sœurs et $Z_{t}$ un coefficient qui exprime l'effet cumulatif de la sélection pendant $t$ générations $\left(Z_{t}=\sum_{r=0}^{t}(1 / 2) R\right)$. 
Cependant, cette équation est basée sur 2 conditions : (1) l'intensité de sélection est faible, (2) le nombre de familles est grand. Ceci n'est pas le cas dans notre expérience, donc nous avons utilisé l'équation de Burrows (1984) qui n'a pas ces restrictions :

$$
\mathrm{N} / \mathrm{Ne}^{\prime}=1+\mathrm{Z}_{\mathrm{t}}^{2}(\mathrm{f}-1)\left(\mathrm{F}_{2}(\mathrm{x} \alpha ; \varrho)-(1-\alpha)^{2} / \mathrm{f} \alpha^{2}\right)
$$

$f$ étant le nombre de familles par lignée et génération, $\alpha$ la proportion sélectionnée, $\varrho=r_{p}$ et $F_{2}(x \alpha ; \varrho)$ la fonction de distribution binormale tabulée par GuPTA (1963). Si le déterminisme génétique du caractère sélectionné ressemble à celui supposé par le modèle infinitésimal, s'il n'y a pas de sélection gamétique ni d'effets de milieu commun alors $N e^{\prime}$ doit être la valeur espérée de $N e$.

Pour pouvoir apprécier s'il y a des changements dans l'effectif génétique des lignées et l'importance de ces changements, les résultats seront comparés à la valeur de l'effectif génétique dans l'hypothèse où les individus sélectionnés sont choisis au hasard parmi les individus mesurés $\left(\mathrm{Ne}^{\mathrm{R}}\right)$. On a calculé $\mathrm{Ne}^{\mathbf{R}}$ à partir de l'équation (4) pour une intensité de sélection nulle $\left(\mathrm{Ne}^{\mathrm{R}}=12,6\right)$.

Le calcul des pourcentages de gènes de la génération $t$ qui proviennent de chaque famille $i$ de la génération 0 peut être effectué au moyen de l'équation :

$$
P_{i t}=\sum_{j=1}^{k} f_{i j t} x_{j t} / \sum_{j=1}^{k} x_{j t}=\sum_{j=1}^{k} f_{i j t} / 6
$$

$P_{i t}$ étant le pourcentage de gènes de la génération $t$ provenant de la famille $i$, $f_{i j t}$ le coefficient de parenté entre la famille $i$ à la génération 0 et la famille $j$ à la génération $t(\mathrm{j}=1,2, \ldots, \mathrm{k})$ et $x_{j t}$ le nombre de frères et sœurs de la famille $j$ à la génération $t\left(\mathrm{x}_{\mathrm{jt}}=20\right)$.

Pour vérifier s'il y a une relation entre les moyennes phénotypiques des parents de chaque famille dans une lignée à une génération donnée et la contribution de cette famille au groupe sélectionné, un test $\chi^{2}$ est réalisé : les valeurs moyennes des parents de chaque famille sont classées en ordre croissant dans chaque lignée. Nous additionnons les 6 valeurs des contributions au groupe sélectionné des familles dont la place dans chaque arrangement est la même. Les résultats précédents sont comparés à une distribution uniforme, toutes les valeurs espérées étant égales à la contribution moyenne des 6 familles, chacune appartenant à une lignée, c'est-à-dire, 12 individus. Par conséquent :

$$
\chi^{2}=\sum_{i=1}^{6} \frac{\left(s_{i}-12\right)^{2}}{12} \text { où } s_{i}=\sum_{j=1}^{6} c_{i j}
$$

$c_{i j}$ étant la contribution au groupe sélectionné de la famille qui occupe la place $i$ dans l'arrangement de la lignée $j$.

\section{Résultats et discussion}

\section{A. Réponse à la sélection}

L'analyse de la réponse à la sélection publiée dans un article précédent (GAllego \& López-FANjul, 1983) indique que presque toute la réponse apparaît à 
la $2^{\circ}$ génération. En plus, l'héritabilité du caractère, les variances phénotypique et génétique sont respectivement réduites dès la $2^{\mathrm{e}}$ génération à $22 \mathrm{p} .100,40 \mathrm{p} .100$ et 7 p. 100 de leurs valeurs originales. Ces résultats permettent aussi de supposer que la variation génétique du caractère sélectionné dans la population originale est déterminée par la ségrégation d'un petit nombre de gènes dont l'effet est important. En effet, les 6 lignées arrivent rapidement au même plateau, caractérisé par la fixation des mêmes allèles pour tous les locus. Cette conclusion coïncide avec l'interprétation des résultats de l'analyse des effectifs génétiques et des pourcentages de gènes.

\section{B. Effectif génétique}

Les valeurs observées de l'effectif génétique des lignées $(\mathrm{Ne})$ sont rassemblées dans le tableau 1.

On peut observer une grande réduction de l'effectif génétique dans les générations 0 et 1 (56 p. 100 de la valeur prévue sans sélection), tandis que dans les générations postérieures $(2-16)$, ce paramètre demeure presque stable à une valeur de 12,3 , ce qui n'est pas significativement différent de la valeur attendue sans sélection $(12,6)$. Le coefficient de régression de $\mathrm{Ne}$ sur le numéro de la génération, $t$, dans la dernière période, est de $0,035 \pm 0,098$, ce qui n'est pas significativement différent de zéro. La période où l'effectif génétique se réduit correspond à celle où la réponse est la plus grande et où les variances phénotypique et génétique additive et l'héritabilité sont réduites aussi drastiquement.

\section{TABleaU 1}

Effectif génétique moyen (Ne) estimé selon l'équation de JAMES (1962) en valeur absolue et en pourcentage de l'effectif génétique prévu sans sélection $(\mathrm{Ne} R)$.

Mean effective size (Ne) estimated through JAMES' equation (1962) in actual values and as a percentage of the effective size expected without selection $\left(\mathrm{Ne} \mathrm{R}^{\mathrm{R}}\right)$.

\begin{tabular}{|c|c|c|}
\hline Génération & $\mathrm{Ne}$ & $\mathrm{Ne} / \mathrm{NeR}(\times 100)$ \\
\hline 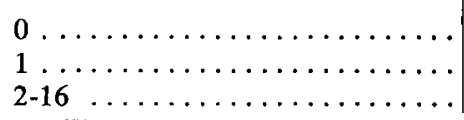 & $\begin{array}{r}7,3 \\
6,7 \\
12,3\end{array}$ & $\begin{array}{l}58 \\
53 \\
98\end{array}$ \\
\hline
\end{tabular}

Ecart-type moyen de $\mathrm{Ne}=3,2$ (calculé avec les 6 valeurs de $\mathrm{Ne}$ par génération dès la $2^{\circ}$ ). Etendue des valeurs de $\mathrm{Ne}$ entre la génération 2 et la $16: 9,9-15,1$.

Mean standard error of $\mathrm{Ne}=3,2$.

Range of $\mathrm{Ne}$ from generation 2 to $16: 9.9-15.1$.

Pour estimer l'effet de la stérilité sur l'effectif génétique, on calcule sa valeur à chaque génération sans tenir compte du remplacement des couples stériles. Les valeurs obtenues montrent que, contrairement à des expériences antérieures (YoO, 1980), il n'y a pas de réduction de l'effectif génétique due à la stérilité, à aucun moment. La valeur moyenne du rapport des effectifs génétiques calculés avec et sans remplacement des couples stériles est égale à 0,9996 . 
On calcule la valeur espérée de l'effectif génétique, $N e^{\prime}$, en utilisant l'équation de Burrows (1984). Les paramètres employés dans l'estimation sont la corrélation phénotypique intra-classe calculée à chaque génération et la valeur moyenne de l'intensité de sélection observée, au lieu de l'héritabilité estimée dans la population originale et l'intensité de sélection théorique, en raison de leur grande réduction pendant l'expérience. Ce changement de paramètres ne permet pas l'utilisation de cette équation pour toutes les générations dès le départ, mais contraint à son application, génération après génération. Les résultats obtenus sont portés dans le tableau 2.

\section{TABLEAU 2}

Valeur de l'effectif génétique moyen estimé selon l'équation de Burrows (1984) (Ne') en valeur absolue et en pourcentage de la valeur prévue sans sélection $\left(\mathrm{Ne} e^{R}\right)$.

Mean effective size estimated through Burrows' equation (1984) (Ne') in actual values and as a percentage of the expected value without selection $(\mathrm{Ne})$.

\begin{tabular}{|c|c|c|}
\hline Génération & $\mathrm{Ne}^{\prime}$ & $\mathrm{Ne}^{\prime} / \mathrm{Ne}^{\mathrm{R}}(\times 100)$ \\
\hline 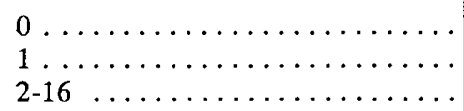 & $\begin{array}{r}8,6 \\
7,6 \\
10,2\end{array}$ & $\begin{array}{l}68 \\
60 \\
77\end{array}$ \\
\hline
\end{tabular}

Etendue des valeurs de $\mathrm{Ne}^{\prime}$ entre la génération 2 et la $16: 7,3-11,4$.

Range of $N e^{\prime}$ from generation 2 to $16: 7.3$ - 11.4.

La valeur de $N e^{\prime}$ est toujours inférieure à 12,6 du fait d'une corrélation phénotypique non nulle, parce qu'elle peut inclure quelques effets de milieu commun. La valeur correspondant aux générations 0 et 1 est considérablement plus petite que celle obtenue dans les générations suivantes, en raison de la réduction de cette corrélation au fur et à mesure que la sélection s'opère. Par ailleurs, la valeur du quotient prédite $\left(\mathrm{Ne}^{\prime} / \mathrm{Ne}^{\mathrm{R}}\right)$ est similaire à celle observée $\left(\mathrm{Ne} / \mathrm{Ne}^{\mathrm{R}}\right)$ dans la $1^{\text {re }}$ période alors que les valeurs prédites dans les générations postérieures sont inférieures à celles observées. Dans la dernière période, il n'y a pas de réponse à la sélection et les valeurs de l'héritabilité et de la variance génétique sont très petites. L'effet Robertson n'a pas lieu de jouer et l'effectif génétique des lignées agit de la même façon que si les individus sélectionnés avaient été choisis au hasard parmi ceux évalués $\left(\mathrm{Ne} / \mathrm{Ne}^{\mathrm{R}}\right.$ $=0,98$ ). Il faut signaler que l'application de la méthode de RoBERTSON (1961) et Burrows (1984) pour prévoir l'effectif génétique, nécessite une ressemblance entre le déterminisme génétique du caractère et celui supposé par le modèle infinitésimal. Dans la plupart des expériences (MCBRIDE \& RobERTSON, 1963 ; JONES, 1969 a et b ; Yoo, 1980), les résultats obtenus sont assez similaires à ceux espérés, mais si la variation du caractère sélectionné est contrôlée par un petit nombre de locus, les valeurs des paramètres génétiques changent très rapidement. Dans ces circonstances, l'équation citée perd sa valeur prédictive et les valeurs prévues sont d'autant plus différentes de celles observées que le changement d'héritabilité du caractère est élevé. 


\section{Coefficient de consanguinité}

Les coefficients de consanguinité observés dans les lignées $\left(F_{t}\right)$ sont calculés à partir des généalogies. Les résultats sont portés sur la figure 1.

Il y a une grande concordance entre les valeurs des différentes lignées, comme pour les autres paramètres analysés dans cette expérience.

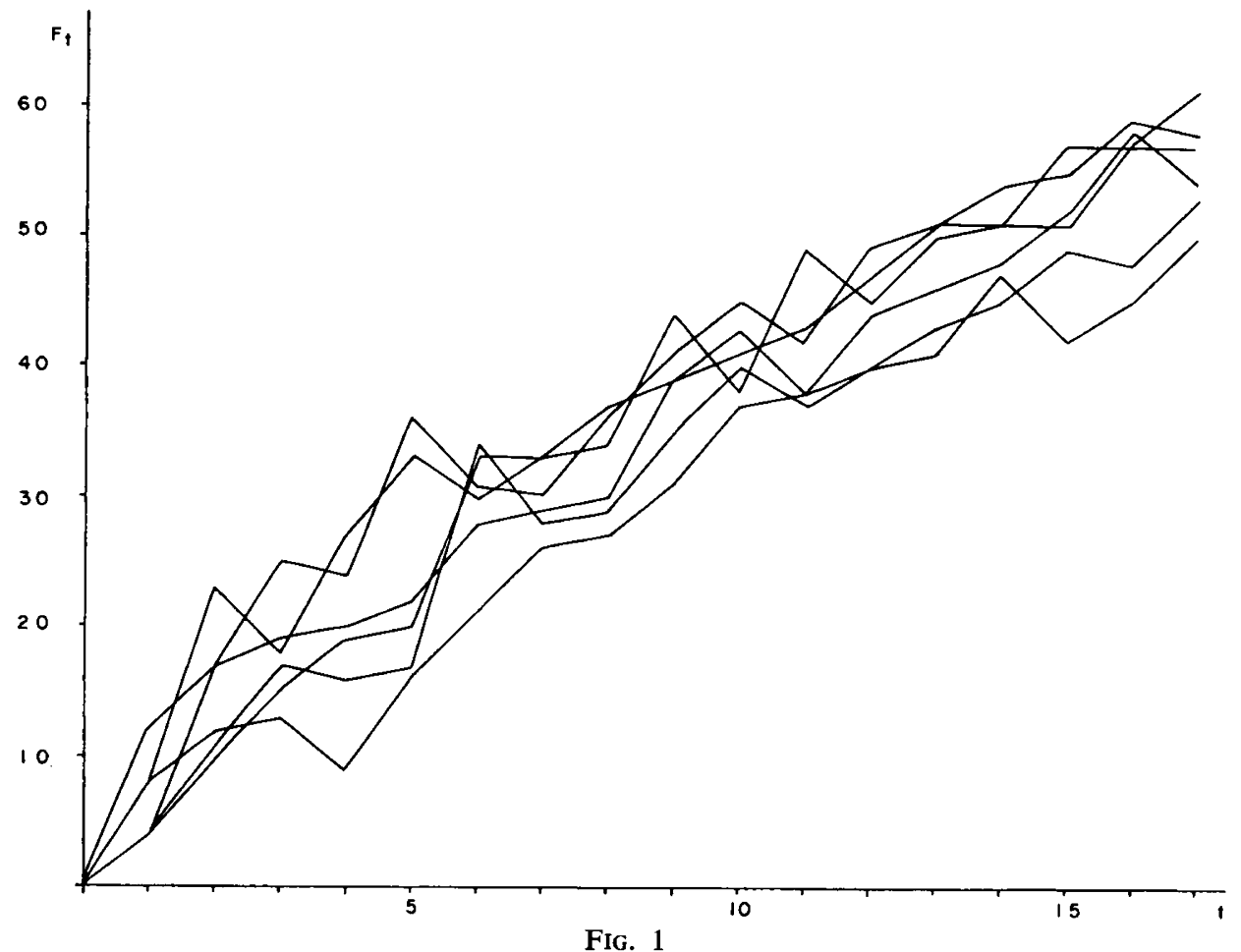

Valeurs de $\mathrm{F}_{\mathrm{t}}$ (coefficient de consanguinité) par génération $(t)$, observées dans les 6 lignées. Values of $F_{t}$ (inbreeding coefficient) by generation ( $t$ ), observed in the 6 lines.

Nous ne pouvons pas obtenir des valeurs de l'effectif génétique dans chaque lignée et à chaque génération en utilisant les variations du coefticient de consanguinité, parce que $F_{t+1}$ n'est pas toujours plus grand que $F_{t}$; cependant, on peut comparer les valeurs moyennes du coefficient de consanguinité avec leur valeur estimée à partir de la méthode de JAMEs (1962). Les résultats obtenus sont portés sur la figure 2. On peut observer que les valeurs obtenues et estimées coïncident presque exactement pendant toute l'expérience. 


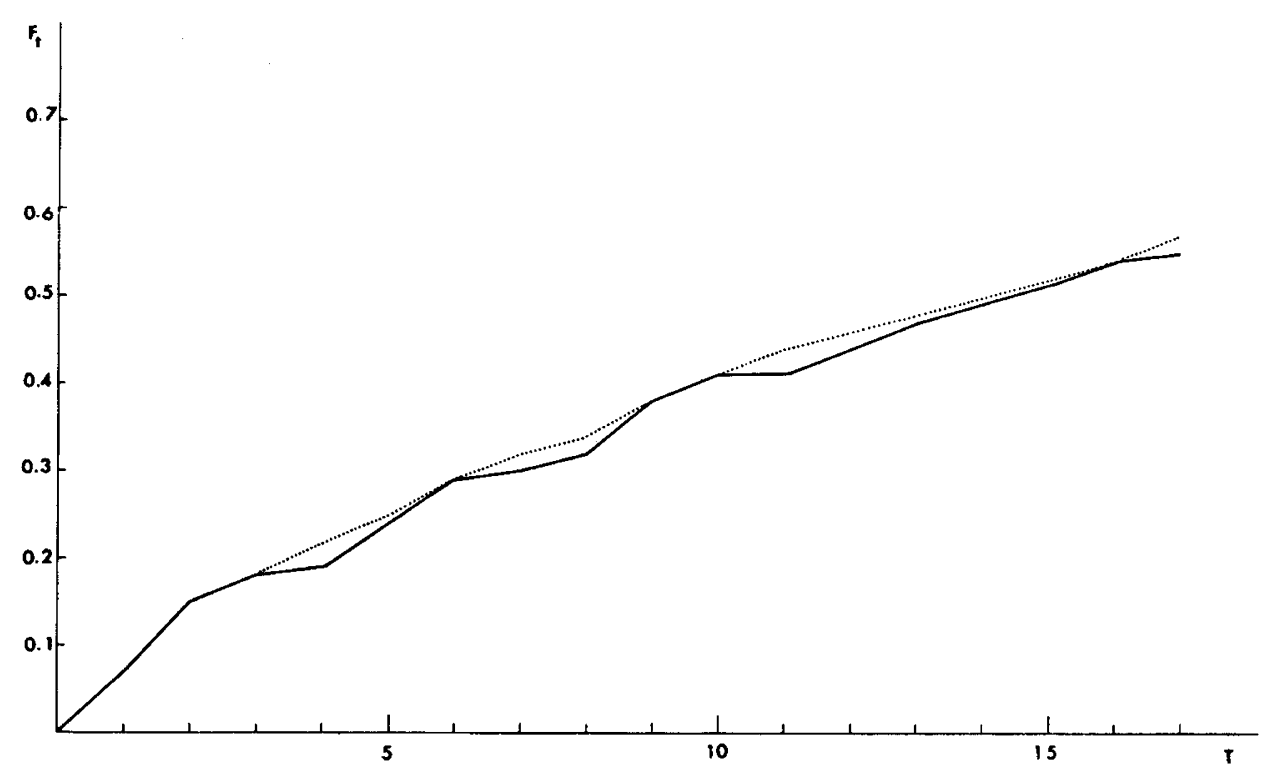

FIG. 2

Le coefficient de consanguinité $\left(F_{t}\right)$ en fonction de la génération $(t)$ : valeur observée moyenne (trait plein)

et valeur estimée à partir de l'effectif génétique observé $\mathrm{Ne}$ (trait pointillé).

Inbreeding coefficient $\left(F_{t}\right)$ by generation $(t):$ mean observed value (solid line) and value estimated using the observed effective size (dotted line).

\section{Pourcentage de gènes}

Dans chaque lignée, on analyse le pourcentage de gènes apportés par chacune des familles qui appartiennent à une génération $t$, dans la population mesurée à chaque génération postérieure $t+k$ (JAMEs \& McBRIDE, 1958). Cette technique est basée sur l'hypothèse que le génotype d'un individu est un échantillon des gènes des ancêtres pris au hasard. Cela signifie qu'il n'y a pas de sélection gamétique.

Quelle que soit la génération initiale considérée $(t)$, il apparaît 2 caractéristiques communes pour toutes les lignées :

a) les familles dont le nombre de descendants s'annule atteignent cette situation en un maximum de 2 générations;

b) les changements importants dans le pourcentage de gènes apportés par chaque famille ont lieu dans les 4 premières générations et après cette période, toutes les familles de chaque lignée ont à peu près la même généalogie.

Pour commencer, considérons la contribution des familles initiales $(t=0)$. Il y a de grandes différences entre les lignées dans la distribution des pourcentages de gènes apportés par chaque famille. Dans 2 lignées, 2 familles fournissent à elles seules les gènes de la population à partir de la $3^{\mathbf{e}}$ génération et une de ces familles 


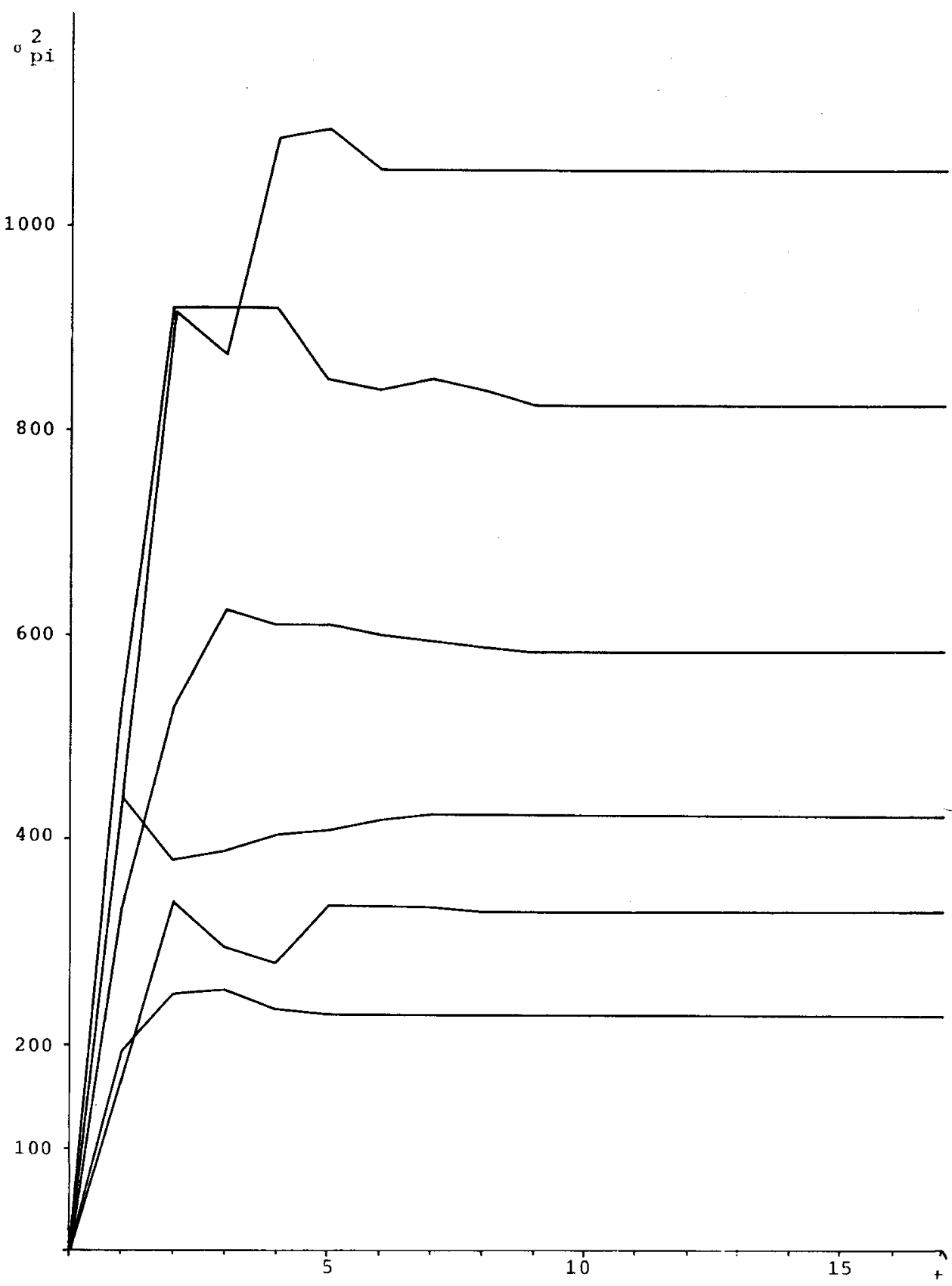

FIG. 3

Variances $\left(\sigma_{P i}^{2}\right)$ des pourcentages de gènes apportés par les familles originales dans les générations suivantes $(t)$, observées dans les 6 lignées.

$$
\text { Variances }\left(\sigma_{P i}^{2}\right) \text { of the percentages of genes }
$$

contributed by the original families in the following generations, observed in the 6 lines. 
apporte, respectivement, dans chaque lignée, 70 p. 100 et 81 p. 100 des gènes; au contraire, dans 2 autres lignées, 4 familles fournissent les gènes dès la $3^{\mathrm{e}}$ génération avec un pourcentage maximum de 43 p. 100 ; ce résultat est attendu dans une expérience où l'on sélectionne intensément un caractère dont l'héritabilité est très grande. La figure 3 montre les variances des pourcentages de gènes apportés par les familles initiales aux différentes générations. Le plus remarquable est qu'à nouveau, presque tous les changements importants ont lieu dans les générations 0 à 2, comme il fallait s'y attendre puisque c'est dans cette période que les changements dans les pourcentages de gènes et les autres paramètres des lignées sont les plus importants. On observe aussi une augmentation de la variance des pourcentages de gènes apportés par les familles initiales, en accord avec RoBertson (1961), due à l'effet cumulatif de la sélection.

Considérons les contributions des familles des générations 3 à 16 : le tableau 3 montre, dans chaque lignée et chaque génération, le nombre de familles dont la contribution au groupe sélectionné et au groupe évalué dans la génération suivante est nulle. On peut observer que leur proportion est plus faible que lors de la période initiale. On peut calculer quelle sera la fonction de probabilité d'une variable qui exprime le nombre de familles ne fournissant aucun gène à la génération suivante, en supposant que ces apports sont établis au hasard. Cette fonction est : $P(0)=0,4403, P(1)=0,4531, P(2)=0,1017, P(3)=4,8 \times 10^{-3}, P(4)=2,84 \times$ $10^{-5}, P(5)=4,6 \times 10^{-7}$. On peut observer que les valeurs les plus grandes, dont la probabilité est infime, ne se retrouvent que dans les générations 0 et 1 , ce qui suggère que la contribution d'une famille ne s'établit pas au hasard dans ces 2 générations.

On cherche s'il y a une relation entre les moyennes phénotypiques des parents de chaque famille et la contribution de cette famille au groupe sélectionné. Cette relation n'existe que lorsqu'il y a une variabilité génétique. Pour cela nous avons réalisé un test $\chi^{2}$ (décrit dans "Matériel et méthodes ») et les résultats indiquent que les contributions sont significativement liées à la moyenne phénotypique des parents dans la $1^{\text {re }}$ génération. Dans les générations suivantes, il n'y a pas une relation significative entre la valeur moyenne des parents d'une famille et leur contribution au groupe sélectionné, c'est-à-dire que ces contributions ne sont pas significativement différentes de celles prévues en considérant seulement l'effet du hasard. Ceci peut indiquer que le caractère sélectionné agit comme si sa variation était fondamentalement non génétique.

Si nous considérons le nombre de familles par lignée et génération qui ne fournissent aucun gène à la génération 17 (tableau 3), la différence entre les 2 premières générations et les autres est bien plus grande que celle observée quand nous considérons la contribution à la génération suivante.

La variance moyenne des pourcentages de gènes apportés par les familles de la génération $t(t=2, \ldots, 16)$ à la génération 17 est 259 (372 et 178 étant le maximum et le minimum, respectivement). Toutes ces valeurs sont inférieures à celles correspondant aux générations 0 et 1 (537 et 441, respectivement).

Par conséquent, on peut envisager que les lignées sont fixées dès la $2^{\mathrm{e}}$ génération, au moins pour les gènes d'effet important. Evidemment ce comportement est très peu probable si le déterminisme génétique du caractère sélectionné est assimilable à celui du modèle infinitésimal. Cela confirme l'hypothèse que la variation initiale du caractère sélectionné est contrôlée dans notre expérience par la ségrégation d'un petit nombre de gènes dont l'effet est très important. 


\section{TABLEAU 3}

Nombre de familles de la génération $\mathrm{t}$ dont la contribution à la génération $\mathrm{t}+1$ est nulle. Les données de la dernière colonne correspondent au nombre total de familles de chaque génération dont la contribution à la génération 17 est nulle.

Number of families in generation $\mathrm{t}$ with null contribution to generation $\mathrm{t}+1$. Data in the last column correspond each generation to the total number of families with null contribution to the 17 generation.

\begin{tabular}{|c|c|c|c|c|c|c|c|c|}
\hline \multirow{2}{*}{ Génération } & \multicolumn{6}{|c|}{ Lignée } & \multirow{2}{*}{ Total } & \multirow{2}{*}{ Total (17) } \\
\hline & 1 & 2 & 3 & 4 & 5 & 6 & & \\
\hline$\ldots \ldots \ldots \ldots$ & 1 & 1 & 1 & 3 & 1 & 3 & 10 & 18 \\
\hline $1 \ldots \ldots \ldots$ & 3 & 2 & 3 & 1 & 3 & 1 & 14 & 15 \\
\hline $2 \ldots \ldots \ldots$ & 1 & 0 & 0 & 1 & 0 & 1 & 3 & 5 \\
\hline $3 \ldots \ldots \ldots$ & 2 & $\mathbf{0}$ & 1 & 2 & 2 & 2 & 9 & 11 \\
\hline $4 \ldots \ldots \ldots$ & 0 & 0 & 2 & 0 & 0 & 1 & 3 & 9 \\
\hline $5 \ldots \ldots \ldots$ & 1 & 0 & 1 & 2 & 2 & 1 & 7 & 7 \\
\hline $6 \ldots \ldots \ldots$ & 1 & 1 & 1 & 1 & 1 & 0 & 5 & 6 \\
\hline$\ldots \ldots \ldots$ & 1 & 0 & 1 & 0 & 1 & 0 & 3 & 7 \\
\hline$\ldots \ldots \ldots$ & 1 & 2 & 0 & 2 & 0 & 2 & 7 & 9 \\
\hline$\ldots \ldots \ldots$ & 1 & 1 & 1 & 1 & 2 & 0 & 6 & 8 \\
\hline $10 \ldots \ldots \ldots$ & 1 & 0 & 1 & 0 & $\mathbf{0}$ & 2 & 4 & 10 \\
\hline $11 \ldots \ldots \ldots$ & 0 & 1 & 1 & 1 & 1 & 0 & 4 & 7 \\
\hline $12 \ldots \ldots \ldots$ & 0 & 1 & 1 & 2 & 1 & 1 & 6 & 8 \\
\hline $13 \ldots \ldots \ldots$ & 0 & $\mathbf{0}$ & 0 & 1 & 0 & 1 & 2 & 4 \\
\hline $14 \ldots \ldots \ldots$ & 0 & 2 & 0 & 0 & 2 & 0 & 4 & 5 \\
\hline $15 \ldots \ldots \ldots$ & 1 & 1 & 0 & 1 & 1 & 0 & 4 & 6 \\
\hline $16 \ldots \ldots \ldots$ & 1 & 1 & 1 & 1 & 1 & 1 & 6 & 6 \\
\hline
\end{tabular}

Il est évident que la technique des pourcentages de gènes (JAMEs \& MCBRIDE, 1958) informe sur la situation des lignées à chaque génération et peut servir de méthode alternative pour le calcul des effectifs génétiques. Ceci peut fournir une représentation plus directe et facile de la situation observée.

Reçu le 28 mars 1985. Accepté le 28 février 1986.

\section{Remerciements}

Nous tenons à remercier le Prof. C. LóPEz-Fanjul pour nous avoir encouragées et pour son aide dans l'élaboration et la discussion des résultats, et aux $D^{\text {rs }}$ M.A. Toro et L. SiLVELA pour leurs commentaires sur le manuscrit.

De même nous remercions Dña. I. Cobos, le $D^{r}$ G. Bolet et M. J.P. Pons pour la révision linguistique.

Ce travail a reçu une aide financière de la «Comision Asesora de Investigacion Cientifica y Técnica ». 


\section{Références bibliographiques}

BURRows P.M., 1984. Inbreeding under selection from unrelated families. Biometrics, 40, 357-366.

Crow J.F., 1954. Breeding structure of populations. II. Effective population number. In : KeMPTHORNE O., BANCROFT T.A., GOWEN J.W., Lush J.L. (ed.), Statistics and Mathematics in Biology, 543-556, Iowa State College Press, Ames, Iowa.

Gallego A., López-Fanjul C., 1983. The number of loci affecting a quantitative trait in Drosophila melanogaster revealed by artificial selection. Genet. Res. Camb., 42 , 137-149.

GUPTA S.S., 1963. Probability integrals of multivariate normal and multivariate t. Annals of Mathematical Statistics, 34, 792-828.

HILL W.G., 1971. Design and efficiency of selection experiments for estimating genetic parameters. Biometrics, 27, 293-311.

HiLl W.G., 1974. Variability of response to selection in genetic experiments. Biometrics, 30, 363-366.

HILl W.G., 1977. Variation in response to selection. Proceedings of the International Conference on Quantitative Genetics, Ames, August 16-21, 1976, PollaK E., KempThorne O., BaIley T.B. (ed.), 343-365, Iowa State University Press, Ames, Iowa.

HiLl W.G., 1985. Effect of population size on response to short and long term selection. Z. Tierzüchtg Zuchtungsbiol., 102, 161-173.

JAMES J.W., 1962. The spread of genes in random mating control populations. Genet. Res. Camb., 3, 1-10.

JAMES J.W., MCBride G., 1958. The spread of genes by natural and artificial selection in closed poultry flock. J. Genet., 56, 55-62.

JONES L.P., 1969 a. Effect of artificial selection on rates of inbreeding in populations of Drosophila melanogaster. I. Effect in early generations. Aust. J. Biol. Sci., 22, 143-155.

JONES L.P., $1969 \mathrm{~b}$. Effect of artificial selection on rates of inbreeding in populations of Drosophila melanogaster. II. Effect of previous selection on rates of inbreeding. Aust. J. Biol. Sci., 22, 157-169.

MCBride G., Robertson A., 1963. Selection using assortative mating in Drosophila melanogaster. Genet. Res. Camb., 4, 356-369.

Robertson A., 1960. A theory of limits in artificial selection. Proc. Roy. Soc. B, 153, 234-249.

Robertson A., 1961. Inbreeding in artificial selection programs. Genet. Res. Camb., 2, 189-194,

WRIGHT S., 1940. Breeding structure of populations in relation to speciation. Am. Nat., 74, 232-248.

Yoo B.H., 1980. Long-term selection for a quantitative character in large replicated populations of Drosophila melanogaster. V - The inbreeding effect of selection. Aust. J. Biol. Sci., 33, 713-723. 